\title{
Difference Field Estimation for Enhanced 3-D Texture Segmentation
}

\author{
E. Ranguelova and A. Quinn \\ Department of Electronic and Electrical Engineering \\ Trinity College, Dublin 2
}

\begin{abstract}
The optimization problem of finding the best match for a thin-plate block of multi-texture 3-D data in a supervised framework is studied in this paper. The textures are modelled as realizations of Gaussian Markov Random Fields (GMRFs)on 3-D lattices. The classification of the central point of the data block is performed by calculating the class probability mass function (p.m.f.s) for the block given the different texture models. The KullbackLeibler measure is proposed for the minimization of the difference between the p.m.f.s distances of the

The three-dimensional (3-D) segmentation of volumetric imagery poses the challenge of estimation and compensation for the existing inter-slice difference within a multi-texture 3-D data. In this paper we propose a novel method to identify the difference field by Kullback-Leibler minimization of the distance between the class probability mass functions (p.m.f.s), calculated at thin-plate 3-D blocks of data, centered at the points of interest. and fast FFT-based technique is presented for calculation of the probability density function (p.d.f.) of the data given the model. This facilitates the calculation of the classification p.m.f.s. in a supervised framework.

The estimated difference field is used to enhance the performance of a computational-volume based 3-D GMRF segmentation algorithm. The performance of the overall method is illustrated with a simulation study of mosaic of synthetic 3-D textures and MRI images of human brain.
\end{abstract}

\section{Introduction}

In many applications (MRI, CT, etc.) the segmentation of volumetric imagery is an important task. The 3-D Markov Random Field (MRF) has been successfully applied for segmenting such 3-D images [1, 2] using region growing approaches.

The 3-D Gaussian Markov Random Fields (GMRFs) has proven a useful model for 3-D textured data [3]. An algorithm we proposed uses a computational volume- based parameter estimation of the model at selected lattice points assuming texture stationarity. The method has the advantages of exploiting the easy implementation of the matrix computations, which follows from the volume geometry. Its drawback is evident when the violation of the above mentioned assumption, is present in regions overlapping data belonging to two or more texture classes. The overlapping along the third dimension can be seen as caused by the shape evolution of different anatomical structures in MRI for instance.

To cope with the problem, difference-compensated computational volume technique has been developed, where adaptation in the temporal direction is implemented in order 
follow the shape evolution [4]. Thus, the texture stationarity assumption is protected, which, in turn, leads to better parameter estimation and the following classification.

In this paper, we propose a new technique for estimation of the difference field present in volumetric data. The method is based on minimizing the Kullback-Leibler distance (KLD) between the class p.m.f.s for 3-D blocks of data, centered on lattice points, candidate for matching. For construction of the p.m.f.s we need to calculate the p.d.f.s of the blocks given the GMRF parameter estimates. This is done using a novel approach for p.d.f. computation, based on 3-D FFT implementation of the computations involving circulant matrices of level three.

The resulting classification scheme is used for evaluation of the shape evolution effect described above and the segmentation is performed after compensation for this effect.

In Section 2 the relevant theory the of 3-D GMRF and the segmentation algorithms are given, followed by presentation of the joint p.d.f. computation in Section 3. The construction of the class p.m.f.s. and the KLD minimization are explained in Section 4. The overall algorithm is tested on mosaics of synthetic 3-D GMRF textures and MRI data and the results are presented in Section 5, followed by conclusions in Section 6 .

\section{3-D GMRF-based Texture Segmentation}

In this work we consider the finite-lattice MRFs. Let $\Omega=\left\{t=(i, j, k) \mid 1 \leq i \leq N_{1}, 1 \leq\right.$ $\left.j \leq N_{2}, 1 \leq k \leq N_{3}\right\}$ be a 3-D rectangular lattice. A 3-D MRF is a set of random variables $\left\{X_{t}, t \in \Omega\right\}, X_{t} \in\{0, \ldots, 255\}$ which represent the gray-level intensities at the points (voxels) of $\Omega$. The MRF is defined in respect to a $q$-th order symmetric neighbourhood geometry $\eta^{q}$, which is determined by the 3-D Maximum Allowable Square (MAS) rule [3]. The random variables satisfy the Markov property, i.e. the conditional probability at a particular voxel depends only on the values of the random field within the neighbourhood of this voxel:

$$
P\left(X_{t} \mid X_{s}, \forall s \in \Omega /\{t\}\right)=P\left(X_{t} \mid X_{s}, \forall s \in \eta_{t}\right),
$$

where the local neighbourhood is defined as $\eta_{t}=\left\{s=t+r, r \in \eta^{q} \mid t, s \in \Omega\right\}$.

The auto-normal model, known as Gaussian MRF (GMRF)[5] has conditional p.d.f, which is normal distribution given by:

$$
\begin{aligned}
& P\left(X_{t} \mid X_{s}, \forall s \in \Omega /\{t\}\right) \propto \\
& \exp \left(-\frac{1}{2 \sigma^{2}}\left\{\left(X_{t}-\mu_{t}\right)-\sum_{s \in \eta_{t}} \theta_{t, s}\left(X_{s}-\mu_{s}\right)\right\}^{2}\right)
\end{aligned}
$$

Here, $\theta_{t, s}$ are the symmetric interaction coefficients (auto-correlation parameters), $\mu$ and $\sigma$ are the conditional mean and variance.

The gray level at voxel $t$ under the GMRF modelling can be represented as linear combination of the gray levels of the neighbouring pixels plus an additive Gaussian noise $E_{t} \sim N\left(0, \sigma_{t}^{2}\right):$

$$
X_{t}=\mu_{t}+\sum_{s \in \eta_{t}} \theta_{t, s}\left(X_{s}-\mu_{s}\right)+E_{t} .
$$

GMRF is a model for homogeneous texture. If the texture is stationary, the parameters are independent of $t$. The model parameters can be estimated by solving the system 
of standard normal equations of form (3), written for each voxel within a rectangular parallelepiped-shaped computational volume $\omega_{t}$, centered on $t$ (see Fig. 1). It is assumed that the texture is stationary within $\omega_{t}$. The solution is a feature vector $\mathbf{f}_{t}=\left(\widehat{\boldsymbol{\theta}}_{t}^{T}, \widehat{\mu}_{t}, \widehat{\sigma}_{t}^{2}\right)^{T}$ assigned to $t$ obtained by Least Squares (LS) technique [3]. This method will be refereed to as the -3D non-compensated or conventional segmentation.

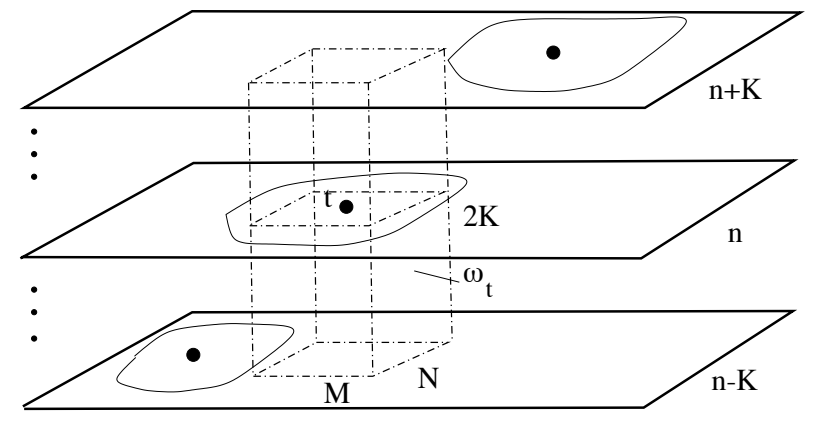

Figure 1: Conventional 3-D processing volumes

The conventional 3-D supervised segmentation has three main stages: learning a codebook of reference feature vectors representing each texture $\mathcal{C}=\left\{\mathbf{c}_{1}, \ldots, \mathbf{c}_{T}\right\}$ ( $T$ is the number of textures), LS estimation and classification in the feature space when a label field $L_{t}=l_{t} \in\{1,2, \ldots T\}$ is inferred. The classification is based on minimization of the distance between each feature vector and the codebook entries. The label assignment is $\widehat{L}_{t}=l_{v}$ such as $l=\arg \min _{i}\left\|\mathbf{c}_{i}-\mathbf{f}_{t}\right\|$ [3]. The label field can be evaluated at all points of $\Omega$ (pixel-by-pixel (PBP)) or at a sparse lattice $\Omega_{R U R}$ as required by a computationally-effective algorithm such as the Recursive Unanimity Rule (RUR) [3]. RUR allows significant accelerations to be achieved in homogeneous regions with the calculations concentrated recursively in the regions of inter-texture boundaries. The method enhances the label connectivity, avoiding the appearance of small isolated regions (holes) in the label field [4].

\subsection{Difference-Field Compensation}

The advantage of the conventional 3-D GMRF-based approach over 2-D GMRF-based independent slice segmentation has been shown in [3]. The method, however, is not robust against violation of the texture stationarity assumption caused by shape evolution of textured object cross-sections along the third dimension. This causes deterioration of the classification accuracy.

To evaluate the shape evolution we estimate a correspondence vector $\mathbf{d}_{t}$, between $t$ and its image $t^{\prime}, \mathbf{d}_{t}=t^{\prime}-t$, which characterizes the inter-slice difference. An estimate of the difference field $\mathbf{D}=\left\{\mathbf{d}_{t}\right\}, t \in \Omega$ allows construction of a compensated computational volume $\omega_{t}^{c}$ (see Fig.2).

Thus the LS estimates are more reliable, since the stationarity assumption is better protected, leading to a significant improvement of the segmentation as shown in $[4,6]$, and in Section 5 of this paper. This method will be referred to as compensated approach.

The overall segmentation framework for the compensated approach is illustrated by the block diagram, shown in Fig.3. 


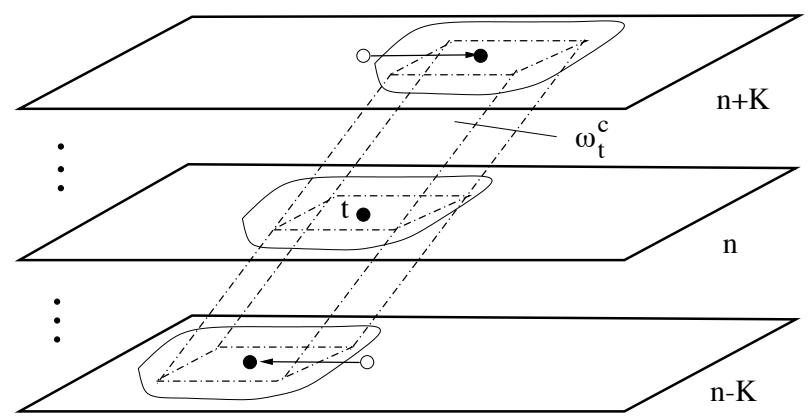

Figure 2: Disparity-compensated (right) 3-D processing volumes

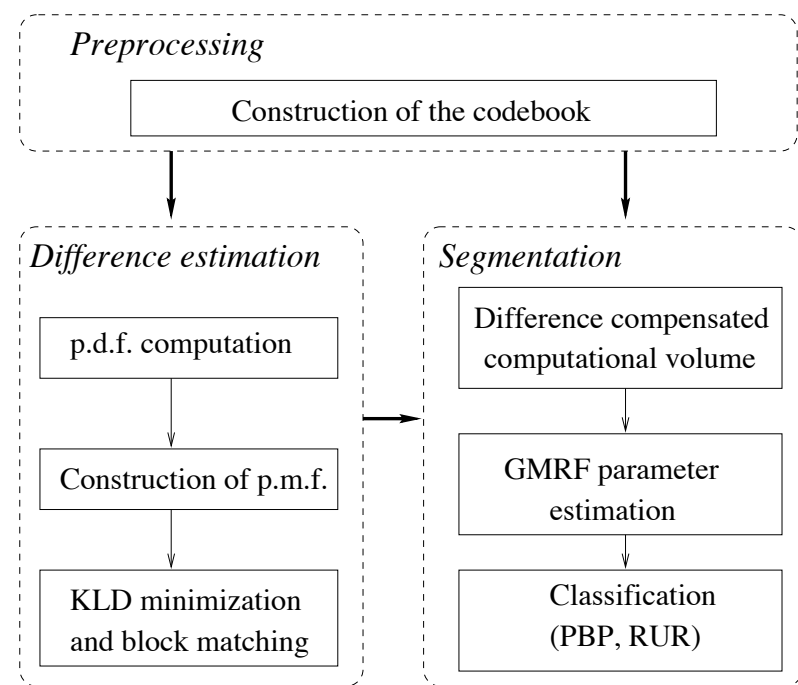

Figure 3: Block diagram of the overall framework

\section{GMRF Joint Probability Computation}

The joint probability of a stationary image $\mathbf{X}=\left\{X_{t}, t \in \Omega\right\}$ of size $n_{1} \times n_{2} \times n_{3}$, may be derived from (3). It is a multivariate normal (MVN) distribution with covariance matrix $\boldsymbol{\Sigma}=\sigma^{2} \mathbf{B}^{-1}[7]$ :

$$
p(\mathbf{x})=\frac{\sqrt{\operatorname{det} \mathbf{B}}}{\sqrt{\left(2 \pi \sigma^{2}\right)^{m}}} \exp \left[-\frac{(\mathbf{x}-\boldsymbol{\mu})^{T} \mathbf{B}(\mathbf{x}-\boldsymbol{\mu})}{2 \sigma^{2}}\right],
$$

$\boldsymbol{\mu}$ is an $m \times 1$ vector of conditional means, and $\mathbf{B}=\left[b_{t, s}\right]$ is the $m \times m$ interaction matrix with elements $b_{t, s}=\delta_{t, s}-\theta_{t, s}$ with $\theta_{t, t}=0$. The necessary and sufficient condition for (4) to be a valid p.d.f. is that $\mathbf{B}$ be symmetric and positive-definite [7]. Such a condition (extended for 3-D GMRFs), dictates that the valid parameter subspace be $\sum|\boldsymbol{\theta}|<0.5$ [8].

Under a toroidal boundary assumption in all dimensions, the $\mathbf{B}$ matrix is a circulant 
f level 3 (block-circulant, which blocks being block-circulant matrices)

$$
\begin{aligned}
\mathbf{B} & =\operatorname{circulant}\left(\mathbf{B}_{1}, \ldots, \mathbf{B}_{k}, \ldots \mathbf{B}_{n_{3}}\right) \\
\mathbf{B}_{k} & =\operatorname{circulant}\left(\mathbf{B}_{1,1}, \ldots, \mathbf{B}_{1, j}, \ldots, \mathbf{B}_{1, n_{2}}\right), \\
\mathbf{B}_{1, j} & =\operatorname{circulant}\left(b_{t, s}\right), t=(i, j, k), s \in \eta_{t} \\
i & =1 \ldots n_{1}, j=1 \ldots n_{2}, k=1 \ldots n_{3} .
\end{aligned}
$$

The $\mathbf{B}$ matrix is sparse and symmetric as well, as a direct consequence of the Markov property and the symmetric neighbourhood structure.

It is known that any circulant and circulant of level 2 matrix can be diagonalized by the 1-D and 2-D unitary DFT respectively $[9,10]$. Similarly a circulant of level 3 can be diagonalized by the 3-D unitary DFT. This leads to an efficient FFT-based algorithm for matrix by vector multiplication. If we consider the $n_{1} \cdot n_{2} . n_{3}$-length vector $\mathbf{x}_{1}=\mathbf{x}-\boldsymbol{\mu}$ obtained by raster-scanning the 3-D block of data $\mathbf{X}_{1}, \mathbf{B}$ be represented by 3-D array $\mathbf{A}(i, j, k)=\left[b_{i, j}^{k}\right]$ of dimensions $n_{1} \times n_{2} \times n_{3}$, the product $\mathbf{x}_{1}^{T} \mathbf{B}$ is equivalent to $3-\mathrm{D}$ circulant convolution of $\mathbf{A}$ and $\mathbf{X}_{1}$ :

$$
\mathbf{X}_{2}(i, j, k)=\sum_{i^{\prime}=1}^{n_{1}} \sum_{j^{\prime}=1}^{n_{2}} \sum_{k^{\prime}=1}^{n_{3}} \mathbf{A}\left(i-i^{\prime}, j-j^{\prime}, k-k^{\prime}\right) \mathbf{X}_{1}\left(i^{\prime}, j^{\prime}, k^{\prime}\right) .
$$

Not that $\mathbf{A}(i, j, k)=\mathbf{A}\left(i \bmod n_{1}, j \bmod n_{2}, k \bmod n_{3}\right)$. As an extension of the 2-D circulant convolution theorem [9], it can be shown that the DFT of the 3-D circulant convolution of two arrays is the product of their DFTs. Using the fast 3-D FFT we can achieve faster multiplication by using the identity:

$$
\mathbf{X}_{2}=\mathcal{F}^{-1}\left(\mathcal{F}\left(\mathbf{X}_{1}\right) * . \mathcal{F}\left(\mathbf{A}_{c}\right)\right),
$$

where $\mathcal{F}=F \otimes F \otimes F$ denotes the 3-D DFT, $\mathcal{F}^{-1}$ - the inverse 3-D DFT , $F$ is the Fourier matrix [10], $\bigotimes$ is the Kronecker product and '*.' means element by element multiplication.

\section{Difference estimation}

Lets $\mathbf{X}$ be a 3-D block of homogeneous GMRF texture and let $C=c \in L=\{1, \ldots, T\}$ be a class discrete random variable taking values among the texture indexes in an ordered set. When a class membership uncertainty exists, one can evaluate the conditional probability (likelihood) of $\mathbf{x}$ given the model class $c$ and its reference parameters $\mathbf{r}_{\mathbf{c}}=\left[\boldsymbol{\theta}_{c}^{T}, \mu_{c}, \sigma_{c}^{2}\right]^{T}$ :

$$
p\left(\mathbf{x} \mid c, \mathbf{r}_{c}\right)=\frac{\sqrt{\operatorname{det} \mathbf{B}_{\mathbf{c}}}}{\sqrt{\left(2 \pi \sigma_{c}^{2}\right)^{m}}} \exp \left[-\frac{\left(\mathbf{x}-\boldsymbol{\mu}_{c}\right)^{T} \mathbf{B}_{\mathbf{c}}\left(\mathbf{x}-\boldsymbol{\mu}_{c}\right)}{2 \sigma_{c}^{2}}\right] .
$$

Eqn. (7) may be evaluated using the algorithm described in Section 3. The conditional probabilities $p\left(\mathbf{x} \mid c, \mathbf{r}_{c}\right)$ evaluated for all classes may be normalized to yield the class p.m.f. $p\left(c, \mathbf{r}_{c} \mid \mathbf{x}\right)$ assuming a uniform prior for $C$. 


\subsection{Kullback-Leibler Distance-based Similarity Measure}

The Kullback-Leibler Distance (KLD) has been successfully used as a texture similarity measure [11]. To find the best match $t^{\prime}$ of the current voxel $t$ among all candidates, we evaluate KLD (or relative entropy) between the class p.m.f.s $p$ and $p^{\prime}$ as:

$$
D\left(p \| p^{\prime}\right)=\sum_{i=1}^{T} p_{i} \log \frac{p_{i}}{p_{i}^{\prime}},
$$

where $T$ is the number of textures present. For our purposes $p$ represents the current, and $p^{\prime}$ - the candidate, site. KLD gives us a measure of similarity between the overall profiles of the class p.m.f.s.

\subsection{Matching}

A 3-D thin-plate volume-based matching technique for estimation of the difference field D is proposed using the ideas above. Consider two 3-D blocks of size $L \times L \times 3$, namely $\mathbf{X}_{t}$ and $\mathbf{X}_{t^{c}}$, centered at $t$ and at a candidate point $t^{c}$ at slice $k^{\prime}$ respectively. Let the associate class p.m.f.s be $p_{t}($.$) and p_{t^{c}}($.$) . The difference vector \mathbf{d}_{t}$ at site $t$ is estimated by a matching procedure, which aims to find the best candidate $t^{\prime}$, minimizing the KLD between the class p.m.f.s, calculated at the two blocks. Hence $t^{\prime}=$ $\arg \min _{t^{c}} K L D\left(p_{t} \| p_{t^{c}}\right)$. The matching is performed by examining candidate blocks in slice $k^{\prime}$ according to a search strategy within a predefined restricted set of candidate points.

\section{Simulation results}

The proposed method has been tested on synthetic images for a two and a three class segmentation problem. Some preliminary results on real MRI data are presented as well.

\subsection{Two class problem}

Two 2 nd order 3 -D textures of size $128 \times 128 \times 11$ were generated using the 3 -D GMRF sythesis algorithm given in [3]. The GMRF parameters were chosen to be similar in the $x, y$ dimensions and different in the $z$ direction, which implies that $z$-going texture correlation will be key disriminants during classification. A texture mosaic volume was created using a ground-truth mask simulating translation of a circular object with displacement of $(+5,+5)$ voxels between adjacent slices. On the first row of Figure 4 the ground truth mask model and the middle slice of the data volume are given.

It can be seen that it is impossible to distinguish visually the two textures. The 2-D segmentation (using 2nd order 2-D GMRF [3]) of the slice is given on the second row, left. The method fails, because the subset of parameters, describing the interaction in the $x-y$ plane are not enough to provide good estimates. The results of the 3 -D conventional (non-compensated) segmentation vs. the compensated methods provided the exact and the estimated difference fields are shown on the second row (left) and on the third row the with a ground truth mask superimposed. The results shown are obtained after using a maximum depth $d=11$ of the computational volume $\omega_{t}$, except for the estimated field segmenation, because the difference field is obtained using 'thin-plate' volumes as 
escribed in 4.2. The figure clearly illustrates the advantages of the 3-D over the 2-D GMRF model as well as the compensated over the conventional approach.

The mean classification accuracy for different depths of the compuational volume are given in Figure 5.
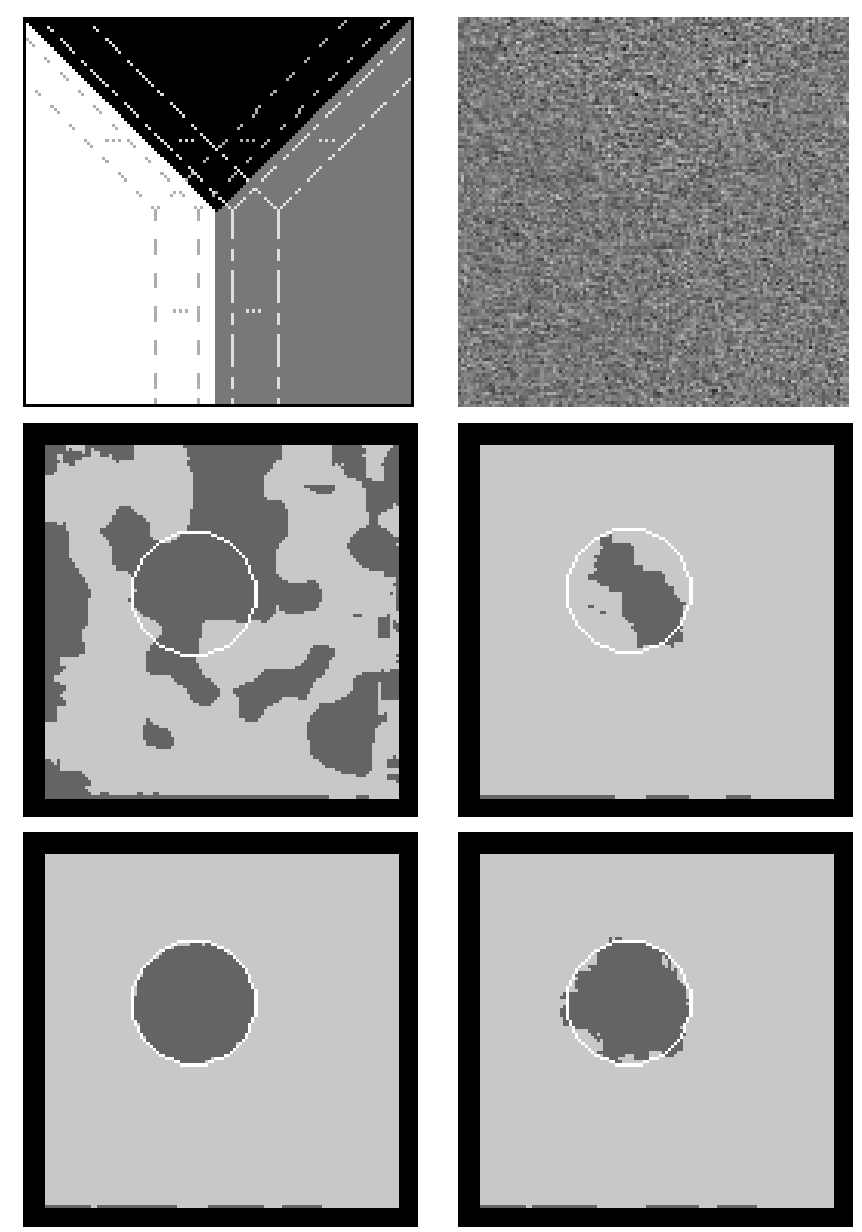

Figure 4: First row: generation mask and slice 6 from synthetic data set; Second row: left:2D and right:3D non-compensated segmentation results with superimposed mask; Third row: 3-D compensated with the known (depth 11) and with the estimated difference (depth 9)

The general trend of the curves shows significant improvement of the classification performance when the difference field is taken into account. Increasing $d$ the degree of stationarity assumption violation increases causing the conventional approach to fail. In contrast, the compensated approach shows better performance due to the more reliable LS estimates, obtained from a bigger stationary texture volume. The compensated segmentation using the proposed approach for calculating the difference field shows a good performance, the error curve tending towards the best possible one. 

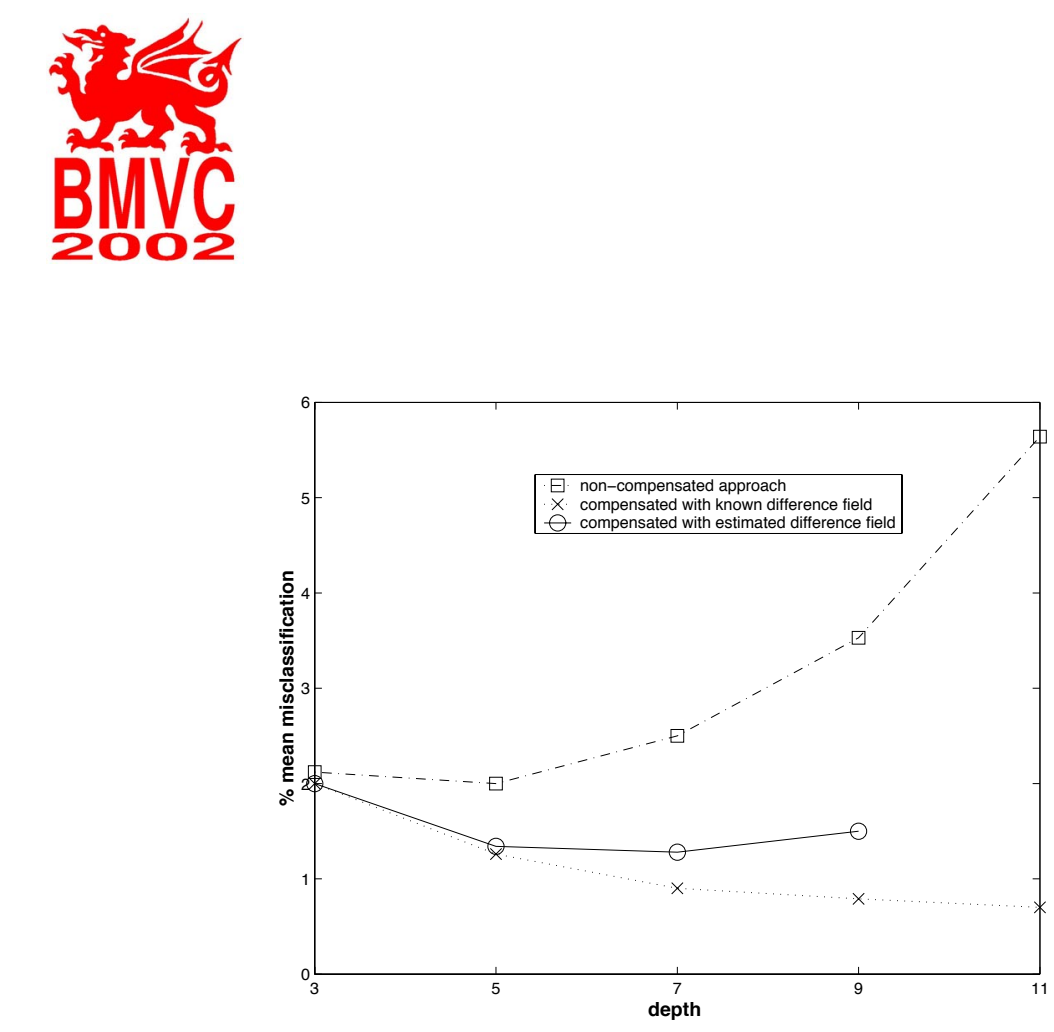

Figure 5: Two class problem: \% mean misclassification for different 3-D segmentation schemes.

\subsection{Three class problem}

More complicated simulation using 3rd order 3-D GMRF and 3 class texture mosaic was performed. The simulations showed that we need to increase the model order $q$ with increasing the number of textures $T$. A higher $q$ provides bigger neighborhood support and hence more $\theta$ parameters which adds extra dimensionality in the feature space. The textures chosen have very similar $x-y$ parameters and differ by the $z$-going correlations. The ground truth mask model, the middle slice of the gray-level data and the segmentation results are illustrated on Figure 6.

The results showed similar performance trends of all compared methods as the two class problem. The percentage of misclassification for the given slice was $8 \%$ for $d=3$ for all 3-D methods, dropping to $0.79 \%$ for the compensated method given the exact difference field, and only to $5.3 \%$ for the compensated one give the estimated field, which is improved only by $0.04 \%$ from the non-compensated method.

In both two and three class problems the proposed minimizing KLD between the class p.m.f.s showed excellent results when classifying homogeneous textures and very good results in overlapping textures regions.

\subsection{MRI}

The segmenataion method has been tested on a subvolume of size $128 \times 128 \times 9$ from a real MRI data sequence of a human brain. In Fig.7, the concsequtive slices of the data set are shown.

In Fig. 8 the main segmentation results for the middle frame of the volume are illustrated. The left image shows the result (depth 9) after non-compensated approach was used. The right image shows the segmentation result $(d=9)$ after the difference field was estimated and compensated for. The better performance of the latter for the maximum depth can be clearly seen. We have noted such improvement in cases of all different 
depths of $\omega_{t}\left(\omega_{t}^{c}\right)$.

\section{Discussion}

In this paper we propose a new FFT-based technique for evaluation the joint 3-D GMRF p.d.f. It proves to be a powerful computational engine for a 3-D texture classification tasks. A complete framework for difference field estimation in a 3-D multi-textured data has been developed. It can be used to enhance significantly a 3-D supervised segmentation scheme and shows very good results on synthetic volumetric images and real real Magnetic Resonance Images (MRI) of a brain.

\section{References}

[1] Soo Choi and Jae Eun Lee et al. "Volumetric Object Reconstruction Using 3D-MRF Model-Based Segmentation". IEEE Transactions on Medical Imaging, 16(16):997$892,1997$.

[2] K. Held and Elena Rota Kops et al. "Markov Random Field Segmentation of Brain MR Images". IEEE Transaction on Medical Imaging, 16(6):878-886, 1997.

[3] E. Ranguelova and A. Quinn. "Analysis and Synthesis of Three-Dimensional Gaussian Markov Random Fields”. In Proc. of ICIP'99, Kobe, Japan, 1999.

[4] E. Ranguelova and A. Quinn. "Displacement-field-compensated Segmentation of 3D Images". In PhD workshop "Cybernetic and Informatics Eurodays",Marianska, Czech Republic, 2000.

[5] Rama Chellappa. “Two-dimensional Discrete Gaussian Markov Random Field Models for Image Processing”. Progress in Pattern Recognition 2, pages 79-112, 1985.

[6] E. Ranguelova and A. Quinn. "Registration Preprocessing for Enhanced 3-D Segmentation". In Proc. of Irish Signals and Systems Conference, Maynooth, Ireland, pages 346-351, 2001.

[7] S.Z.Li. "Markov Random Field Modeling in Computer Vision”. Springer-Verlag, 1995.

[8] S. Lankshmanan and H. Derin. "Valid Parameter Space for 2-D Gaussian Markov Random Fields". IEEE Transactions on Information Theory, 39(2):703-709, 1993.

[9] Anil Jain. “Fundamentals of Digital Image Processing”. Prentice Hall, 1989.

[10] P. Davis. “Circulant matrices”. John Wiley and sons, 1979.

[11] Minh Do and Martin Vetterli. "Texture Similarity Measurements using KullbackLeibler Distance on Wavelet Subbands". In Proc. of ICIP'2000, Vancouver, Canada, 2000 . 

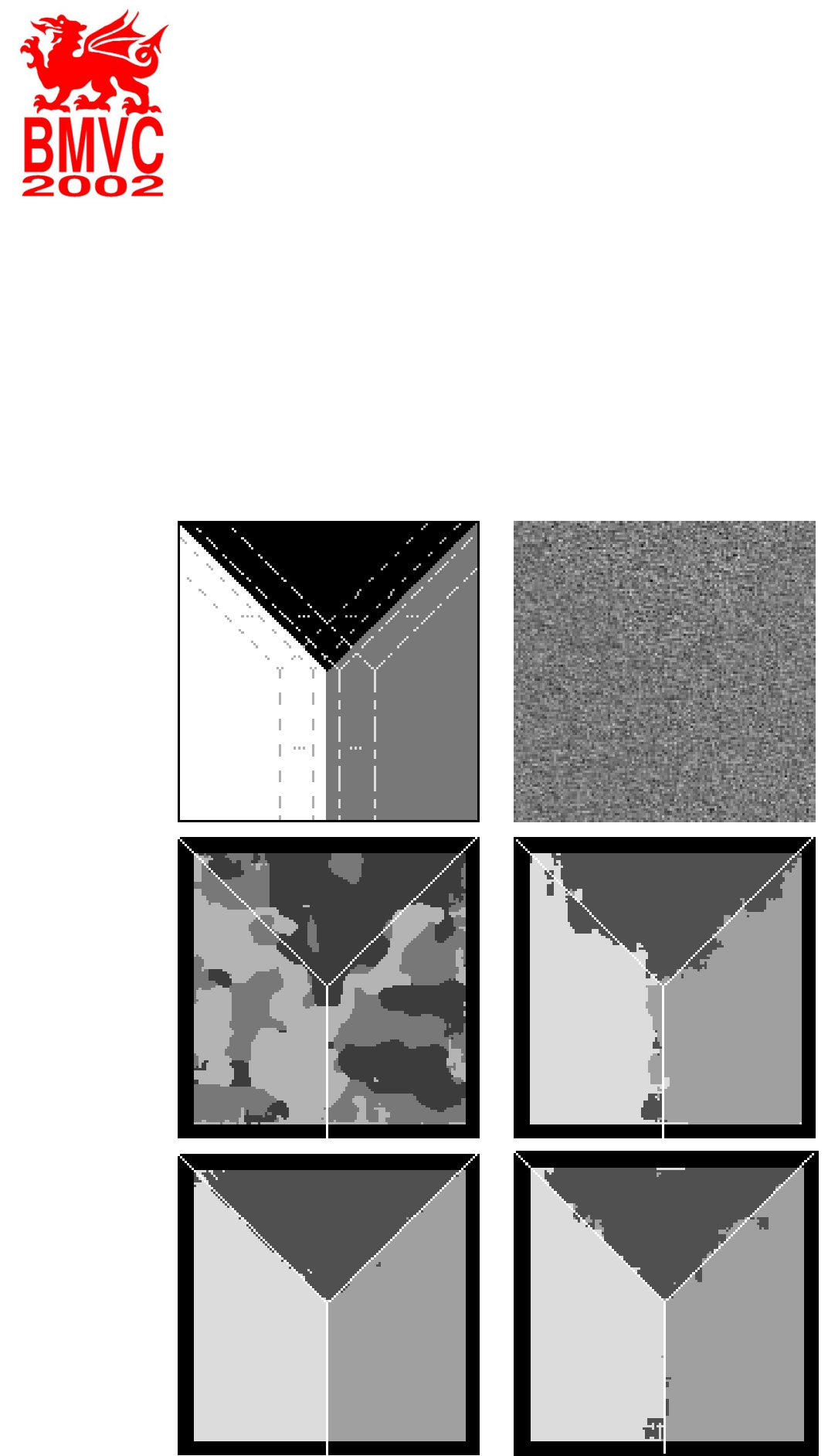

Figure 6: First row: generation mask and slice 6 from synthetic data set; Second row: 2D and 3D non-compensated segmentation results with superimposed mask; Third row: 3-D compensated with the known (depth 11) and with the estimated difference (depth 5) 

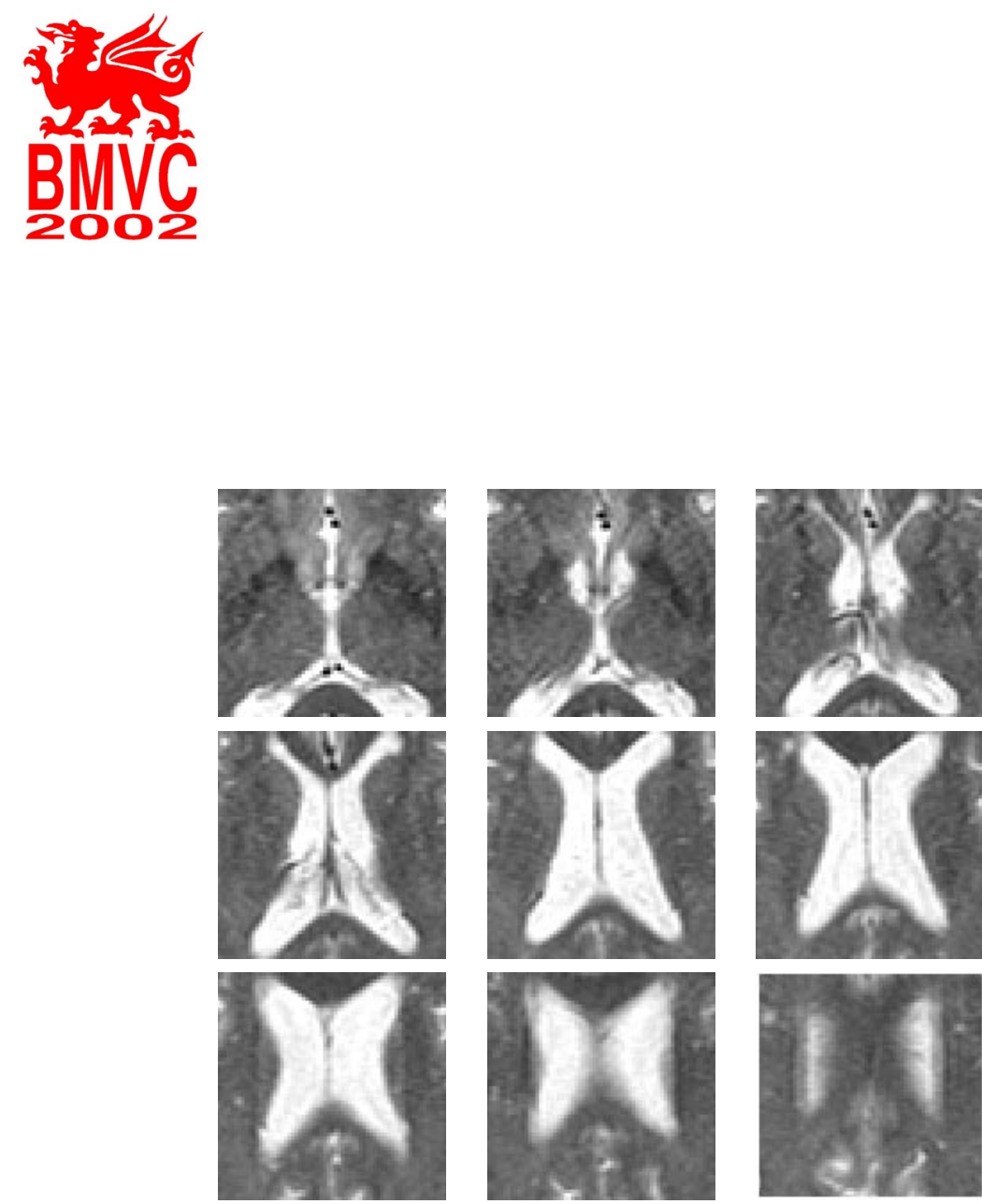

Figure 7: The slices of the MRI sequence
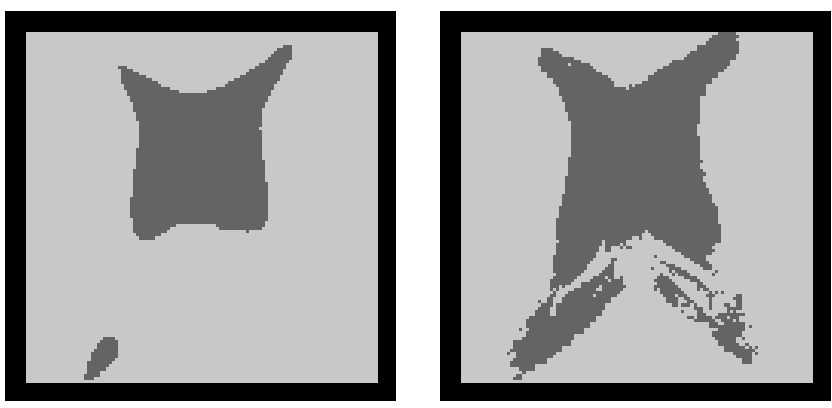

Figure 8: Left: 3D non-compensated result for the middle slice, depth: 9; Right: 3D compensated result, depth: 9; 